\title{
Cosecant-Squared Radiation Pattern Surface Wave Antenna For Millimeter-wave FMCW Vertical- Looking Radar System
}

\author{
Jiashu Yang \\ Department of Electronic and Electrical Engineering \\ University College London \\ Torrington Place, London WC1E 7JE, United Kingdom \\ uceejy1@ucl.ac.uk
}

\author{
Kin-Fai Tong \\ Department of Electronic and Electrical Engineering \\ University College London \\ Torrington Place, London WC1E 7JE, United Kingdom \\ k.tong@ucl.ac.uk
}

\begin{abstract}
This work presents our preliminary work on an electric field (E-field) prediction technique and near-field to far-field transformation of a surface wave antenna with a cosecant-squared pattern for a millimeter-wave FMCW vertical-looking radar system. Fourier and Gaussian fitting models were used to predict the magnitude and phase of E-field on the antenna surface, and the prediction error at the center operating frequency of $34.5 \mathrm{GHz}$ are $4.6 \%$ and $4.6^{\circ}$ respectively. The far-field E-plane pattern was achieved by applying Fourier Transform to the predicted nearfield E-field distribution.
\end{abstract}

Key words - Surface-wave antennas, cosecant-squared pattern, electric field (E-field) prediction, near-field to far-field transformation.

\section{INTRODUCTION}

Trapped Surface Wave (TSW) is the type of surface wave we utilized in this study, it propagates along a dielectric coated planar conductor without radiation [1], [2]. Different launchers, such as waveguide horns [3] or dipole arrays [4], [5], have been proposed to excite a surface wave. The conventional planar surface wave structure with stable surface reactance guides energy along its surface without radiation, in order to induce radiation from the surface, the surface impedance must be varied either by a sudden termination of the guiding structure or an abrupt junction with a different reactive surface [6]. The patterns radiated by surface wave antennas are usually in the shape of a fan-beam and their gain normally does not exceed 20 dBi. Frequency coverage of surface wave antennas extends from very high frequency (VHF) band to millimetre-wave frequency band [7].

In our recent FMCW vertical-looking radar (VLR) system design [8], an antenna with low profile and cosecant-squared radiation pattern is required to offer the portability and improvements for the radar data processing. Cosecant-squared pattern enable an adapted distribution of the radiation in beam pattern and thus useful in applications where a uniform power flux illumination at the same altitude is required [9]. It is because the radiation pattern can provide a more stable signal strength as a target moves with a constant height within the beam. Conventionally, such antennas are utilized in air- surveillance radar systems to detect an approaching target at a constant height with constant power which means that the received power is in dependent of the radar range. There are a few techniques to form a cosecant squared pattern, including curve shaped reflector [10], phased array antenna [11], shaped lens antenna [12] and reflector array [13].

A planar 2D millimeter-wave surface wave antenna based on [14], [15] and the partially reflective screen (PRS) technique is proposed in this work. The full 3D electromagnetic simulation was first performed and the near E-field data (magnitude and phase) was extracted from CST Studio Suite 2019 [16]. The Efield data have been studied and predicted by Fourier and Gaussian curve fitting model [17]. The near-field E-field is then transformed to far-field to predict the radiation pattern.

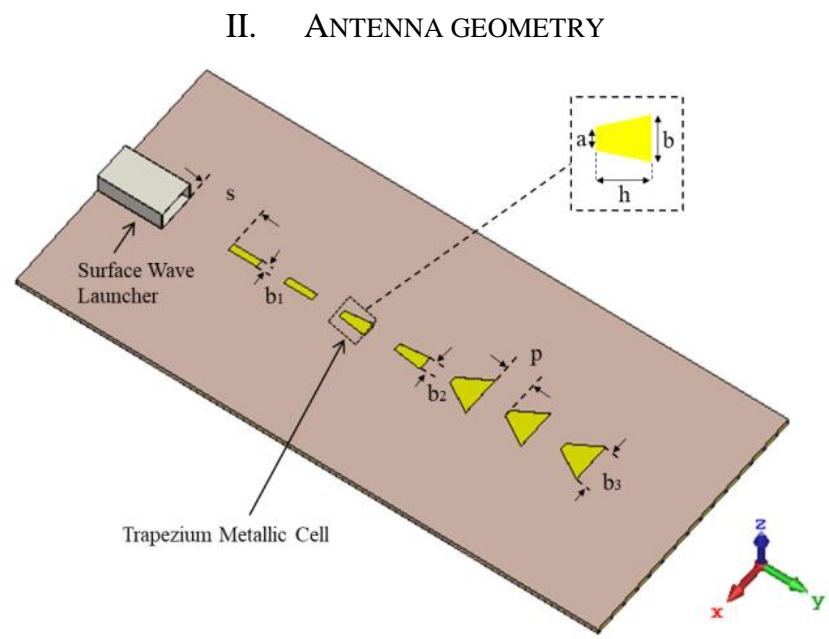

Fig. 1. The proposed surface wave antenna.

The geometry of the proposed surface wave antenna operating at $34.5 \mathrm{GHz}$ is shown in Fig. 1. The antenna consists of a ground plane, a piece of dielectric substrate, seven trapezium metallic cells and a surface wave launcher. The seven trapezium metallic cells have different physical parameters. The short edges (a) of the seven trapezium metallic cells are same with length $0.125 \lambda_{0}$ while the long edges (b) have three different lengths, i.e. $\mathbf{b}_{\mathbf{1}}=$ $0.125 \lambda_{0}, b_{2}=0.25 \lambda_{0}$ and $b_{3}=0.75 \lambda_{0}$ respectively. The height (h) of seven trapezium cells are $0.5 \lambda_{0}$ in the proposed 
antenna. The separation from the front edge of the first metallic cell to the aperture of the surface wave launcher is $\boldsymbol{\lambda}_{\mathbf{0}}$. The spacing (p) between the cells is $0.25 \boldsymbol{\lambda}_{\mathbf{0}}$. Rogers 5880 microwave substrate of dielectric constant $\left(\boldsymbol{\varepsilon}_{\mathbf{r}}\right)$ equals 2.2 , thickness equals $0.762 \mathrm{~mm}$ and loss tangent $(\tan \boldsymbol{\delta})$ equals 0.009 at $10 \mathrm{GHz}$ is used; it will provide the surface impedance with the appropriate excitation efficiency of the surface wave launcher [15].

\section{RESULTS}

By setting an array of field monitors at the height of $1 \mathrm{~mm}$ above the cells along the $y$-axis, the magnitude and phase of the E-field of the surface wave antenna are extracted from CST. When the wave propagates through the surface, the magnitude and phase of the E-field have different changing patterns at different position relative to the cell, as shown in Fig. 2 and Fig. 3. This variation pattern through each cell can be divided into four zones, which are enter zone, cell zone, exit zone and surface wave (SW) zone. The E-field variation in the four zones is studied by using different order of Fourier and Gaussian curve fitting model. Equation (1) and (2) show the lowest order of the fitting model. Then the model can be used to predict the phase and magnitude of the E-field in near-field.

$$
\begin{gathered}
f(x)=a_{0}+a_{1} \cos (\omega x)+a_{2} \sin (\omega x) \\
g(x)=c_{1} e^{-\left(\frac{x-c_{2}}{c_{3}}\right)^{2}}
\end{gathered}
$$

Where $a_{1}, a_{2}, a_{3}, c_{1}, c_{2}$ and $c_{3}$ are the curve fitting parameters.

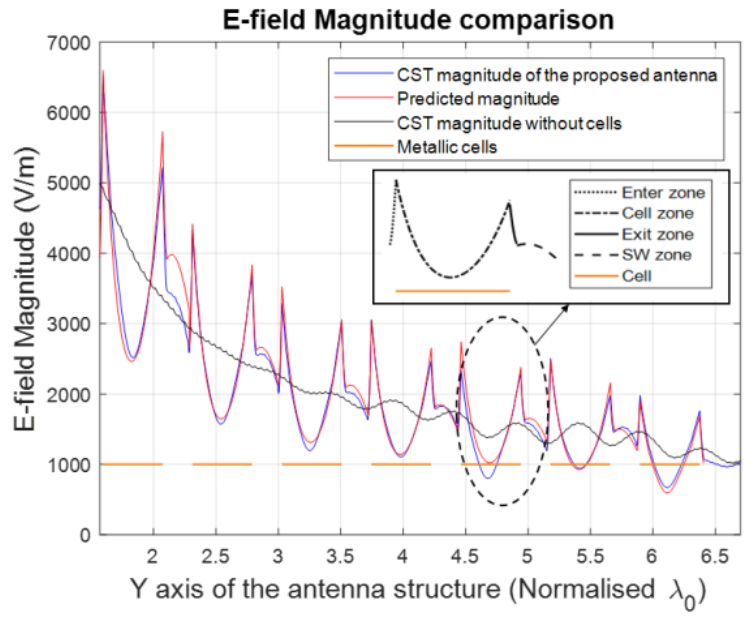

Fig. 2. Magnitude comparison between prediction and CST at $34.5 \mathrm{GHz}$.

The comparisons of magnitude and phase of the E-field at $34.5 \mathrm{GHz}$ are shown in Fig. 2 and Fig. 3 respectively. The magnitude and phase of the surface wave antenna without the cells are also presented in the figures for comparison; the variation of magnitude and phase in the four zones can then be easily observed. In Fig. 2, the magnitude has a sudden rise in the enter zone and has a drop in the exit zone. In the surface wave zone, the magnitude tends to follow the changing trend of the magnitude of the surface wave launcher structure. In Fig. 3, the phase changes rapidly in the enter and exit zone. While in the surface wave zone, the phase change is getting slower and follows the phase change of standard surface wave. In this prediction, a span of $2 \mathrm{GHz}$ bandwidth, which is similar to the operating frequency band of the VLR, is chosen to evaluate the prediction performance. The errors of magnitude and phase prediction are shown in Fig. 4. The prediction error of magnitude is $4.6 \%$ while the phase prediction error is $4.6^{\circ}$ at $34.5 \mathrm{GHz}$.

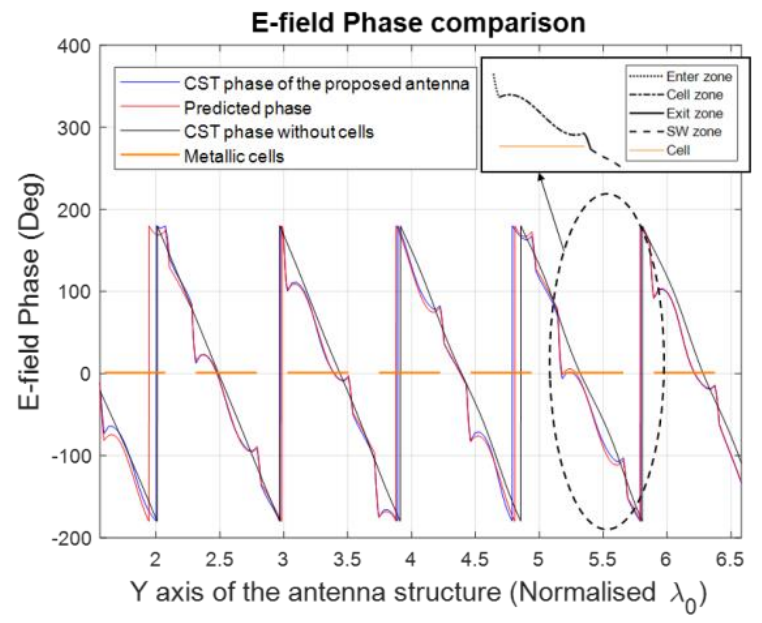

Fig. 3. Phase comparison between prediction and CST at $34.5 \mathrm{GHz}$.

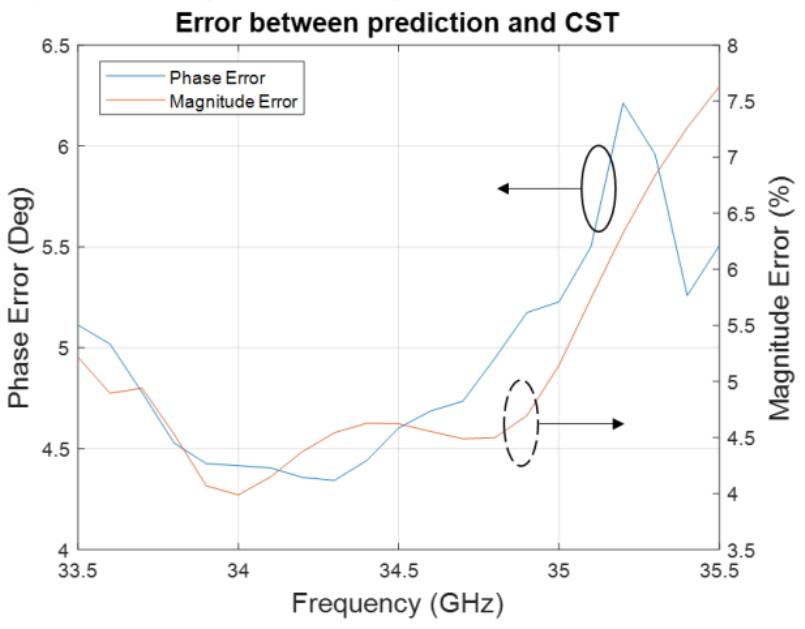

Fig. 4. Error of the prediction.

\section{DISCUSSTION}

The near-field E-field has been studied and predicted based on the trapezium metallic cells in the proposed antenna. Different shapes and sizes of the trapezium metallic strip show different near zone E-field distributions. By varying the size and the shape of the cells, the near-field E-field under different cases can be predicted as well. Based on the predicted E-field and physical designs, an innovative approach to build the relation between them becomes possible by introducing a machine learning program. It will save the time to run multiple simulations of the whole antenna geometry in the 3D full-wave electromagnetic software. Based the machine learning program, the near zone E-field of a physical design can be predicted. And then with the help of equation (3) from [9], the required far field pattern can be obtained through the near-field to far-field transformation (NF2FF). Since the wave spectrum and E-field form a transform pair, which means the far-field pattern can be calculated by applying Fourier transform. 


$$
E(x, y, z)=\frac{1}{4 \pi^{2}} \int_{-\infty}^{\infty} f\left(k_{x}, k_{y}\right) e^{-j k r} d k_{x} d k_{y}
$$

Where $f\left(k_{x}, k_{y}\right)$ represents the wave spectrum of the field, $k_{x}$ and $k_{y}$ are components of propagation factor.

In [8], a cosecant-squared radiation pattern is required to make the practical adjustment easier. According to the required pattern, the near-zone E-field can be calculated by applying farfield to near-field transformation. The desirable physical design of the required pattern antenna can then be achieved.

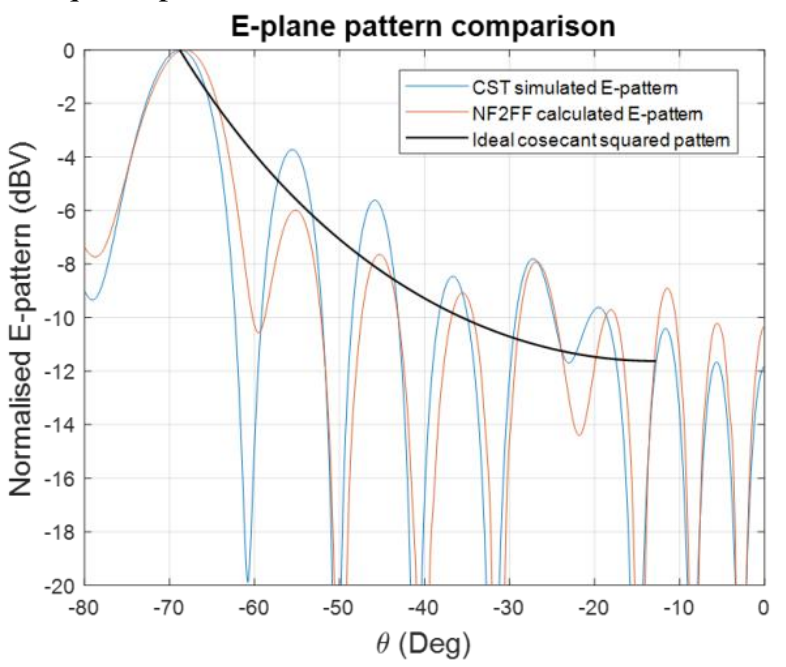

Fig. 5. E-plane pattern at $34.5 \mathrm{GHz}$.

In Fig. 5, the radiation pattern of the proposed near-field to far-field predicted technique is shown. It is compared with the ideal cosecant squared pattern and that provided by CST. The main lobe of NF2FF calculated pattern is consistent with the CST simulation. The discrepancy remains small within the area of $\theta$ from $-80^{\circ}$ to $0^{\circ}$, while the rest has the similar trend. The mismatch of the side lobes caused by the algorithm needs to be improved in later work. The grating lobes will be improved by revising the antenna structure. Machine learning algorithm will be introduced to predict the predicted far-field distribution closer to the ideal cosecant-squared pattern.

\section{CONCLUSION}

The E-field in the near field region and the radiation pattern of the proposed antenna has been predicted by using Fourier and Gaussian mathematical model and Fourier transform with the acceptable error $4.6 \%$ in magnitude and $4.6^{\circ}$ in phase at 34.5 GHz. E-field prediction under different metallic shapes and sizes will be investigated. And machine learning program will be introduced to define the relation between E-field and physical design in the future work.

\section{REFERENCES}

[1] H. M. Barlow and A. L. Cullen, 'Surface waves', Proc. IEEE - Part III
Radio Commun. Eng., vol. 100, no. 68, pp. 329-341, 1953.

[2] T. K. Sarkar, M. N. Abdallah, M. Salazar-Palma, and W. M. Dyab, 'Surface Plasmons-Polaritons, Surface Waves, and Zenneck Waves: Clarification of the terms and a description of the concepts and their evolution', IEEE Antennas Propag. Mag., vol. 59, no. 3, pp. 77-93, 2017.

[3] Z. Shafiq, M. Kuznetcov, V. G. G. Buendia, D. E. Anagnostou, and S. K. Podilchak, 'A Planar Horn Antenna for TM Surface Wave Launching using Substrate Integrated Waveguide Technology', 13th Eur. Conf. Antennas Propagation, EuCAP 2019, no. EuCAP, pp. 2019-2021, 2019.

[4] J. E. Turner, M. S. Jessup, and K. F. Tong, 'A novel technique enabling the realisation of $60 \mathrm{GHz}$ body area networks', Proc. - BSN 2012 9th Int. Work. Wearable Implant. Body Sens. Networks, no. 1, pp. 58-62, 2012.

[5] Ö. A. Civi and P. H. Pathak, 'Array guided surface waves on a finite planar array of dipoles with or without a grounded substrate', IEEE Trans. Antennas Propag., vol. 54, no. 8, pp. 2244-2252, 2006.

[6] L. Felsen, 'Radiation from a tapered surface wave antenna', IRE Trans. Antennas Propag., vol. 8, no. 6, pp. 577-586, 1960.

[7] J. L. Volakis, Antenna Engineering Handbook, 4th ed. McGraw-Hill Education, 2007.

[8] J. Yang, K. F. Tong, K. S. Lim, A. Reynolds, and C. Rawlings, 'Development of Millimeter-wave FMCW Vertical-looking Entomological Radar System', 2019 IEEE Int. Work. Electromagn. Appl. Student Innov. Compet. iWEM 2019, pp. 1-2, 2019.

[9] Constantine A. Balanis, Antenna Theory Analysis and Design. John Wiley \& Sons, Ltd., 1982.

[10] A. Brunner, 'Possibilities of Dimensioning Doubly Curved Reflectors for Azimuth-Search Radar Antennas', IEEE Trans. Antennas Propag., vol. 19, no. 1, pp. 52-57, 1971.

[11]R. S. Elliott and G. J. Stern, 'A new technique for shaped beam synthesis of equispaced arrays', IEEE Trans. Antennas Propag., vol. 32, no. 10, pp. 1129-1133, 1984.

[12] R. Sauleau and B. Barès, 'A complete procedure for the design and optimization of arbitrarily shaped integrated lens antennas', IEEE Trans. Antennas Propag., vol. 54, no. 4, pp. 1122-1133, 2006.

[13] M. Arrebola, J. A. Encinar, and M. Barba, 'Multifed printed reflectarray with three simultaneous shaped beams for LMDS central station antenna', IEEE Trans. Antennas Propag., vol. 56, no. 6, pp. 1518-1527, 2008.

[14] J. Wan, K. F. Tong, and C. Wu, 'The excitation efficiency of surface waves on a reactive surface by a finite vertical aperture', IEEE Antennas Propag. Soc. AP-S Int. Symp., vol. 2015-Octob, no. 1, pp. 1634-1635, 2015.

[15] J. Wan, K. F. Tong, and C. H. Chan, 'Simulation and experimental verification for a $52 \mathrm{GHz}$ wideband trapped surface wave propagation system', IEEE Trans. Antennas Propag., vol. 67, no. 4, pp. 2158-2166, 2019.

[16] CST Studio Suite 2019, 'CST Microwave Studio'. Dassault systemes.

[17] Matlab 2019b, 'MATLAB and Curve Fitting Toolbox Release 2019b'. The MathWorks Inc., Natick. 